\title{
Action Mapping Analysis of Interventional Surgery Robot Based on Master-slave Operation Xue YANG ${ }^{1 *}$, Zhao-Cui HAN ${ }^{2}$, Hong-Bo WANG ${ }^{3}$ \\ ${ }^{1}$ University of Jinan \\ ${ }^{2}$ Parallel Robot and Mechatronic System Laboratory of Hebei Province \\ ${ }^{3}$ Yanshan University, Qinhuangdao \\ me_yangx@ujn.edu.cn \\ ${ }^{*}$ Corresponding author
}

Keywords: master-slave, minimally invasive surgery, operational mapping, graph transformation.

\begin{abstract}
Minimally invasive vascular interventional surgical robot system includes two parts, the master manipulator and the slave end-effector. With the operation of the system need to map the operation of the main hand to the end effector, to achieve master-slave operation. Based on the graph transformation mechanism, this paper analyzes the operation mapping of the two actions of the robot system to twist and deliver the guide wire. That is, first of all to identify the master operating instructions, and the instructions through the graphics transform to get the corresponding action of the slave end effector, to achieve the master and slave operation and lay the foundation for the realization of interventional telesurgery.
\end{abstract}

\section{Introduction}

Nowadays, surgical robots are widely used in many surgical subspecialties, such as laparoscopic surgery, urology, gynecology, orthopedics, and ophthalmic surgery ${ }^{1-3}$. ROBOT systems for minimally invasive surgery (MIS) have been developed by many research groups in order to overcome some disadvantages of MIS such as inconsistent hand-eye coordination and the fulcrum effect of surgical instrument ${ }^{4-6}$.

Vascular interventional surgery is the Digital Subtraction Angiography (DSA) to guide the doctor to insert the catheter, guide wire, saccule and stent into blood vessels and cure lesions so that the catheter reaches the embolism and malformed vascular to dissolve the clot of narrow blood vessels or to use other purposes ${ }^{7,8}$.

Robotic systems and technologies have made remarkable contribution to the innovation enabling various innovative devices and procedures including robotic laparoscopic surgery assist system ${ }^{9}$. The possibility of consultation diagnosis, treatment, and medical intervention from a distance, may greatly impact the quality of life of patients located in isolated areas where access to specialized medical services is limited ${ }^{10}$. Vascular intervention using master-slave surgical robot system, make the doctor away from surgery to avoid radiation. In order to push a guide wire into target branch vessel, the catheter motion inside a insertion trocar port can be described with two degrees of freedom (DOFs), linear and rotational displacement ${ }^{11}$.

A master-slave system is the most popular used in telesurgery system and mainly consists of two parts: a master acting as the controller which is responsible for interaction with human and command the slave, and a slave which is commanded by the master to actually complete the task ${ }^{12}$. Hence the translational and rotational relationship between the master control handles and the slave end effectors should be carefully designed so as to maximally ensure the hand-eye-coordination of the surgeon ${ }^{13,14}$.

\section{Mapping matrix}

Therefore, two actions of the rotation and movement of the mapping mechanism should be studied, that is, to find the corresponding angle mapping matrix and displacement mapping matrix. 
First, the master hand and the two coordinate system $\mathrm{o}_{\mathrm{m}}-\mathrm{xyz}$ and $\mathrm{o}_{\mathrm{s}}-\mathrm{xyz}$ are established (shown in Fig.1), and the coordinate transformation matrix between the two coordinate axes is

$$
T_{0}=\left[\begin{array}{rrrr}
1 & 0 & 0 & 0 \\
0 & 1 & 0 & 0 \\
0 & 0 & 1 & 0 \\
-x_{m} & -y_{m} & -z_{m} & 1
\end{array}\right]
$$

There, $x_{\mathrm{m}}, y_{\mathrm{m}}, z_{\mathrm{m}}$, respectively, indicates that the coordinates of the origin of the master in the slave coordinate system.

1:1Angle Mapping. In order to make the calculation simple and rapid, it is consistent with the direction of the main end coordinate system and the coordinate axes. So as to avoid the rotation transformation in the process of coordinate transformation. For the angle mapping, the transformation is between the two coordinate systems. And around the rotation of any axis, the axis should be translated to a coordinate axis, and then rotate it to be coincidence with the coordinate axis, and finally the corresponding rotation angle transform.

For example: in order to make the rotation axis $l_{\mathrm{m}}$ and $\mathrm{y}_{\mathrm{s}}$ coincide. First of all, $l_{\mathrm{m}}$ should be translate to $l s$, and then rotate around the $z_{s}$ axis to the $\mathrm{y}_{\mathrm{s}} \mathrm{O}_{\mathrm{s}} \mathrm{z}_{\mathrm{s}}$ plane, around the $\mathrm{x}_{\mathrm{s}}$ axis rotation, and eventually coincide with the $\mathrm{y}_{\mathrm{s}}$ axis, and then carry out angle transformation, to transform the position after required by the corresponding inverse transformation will transform the axis of rotation to the original position, the transformation matrix can be expressed as:

$$
T_{1}=\left[\begin{array}{cccc}
1 & 0 & 0 & 0 \\
0 & 1 & 0 & 0 \\
0 & 0 & 1 & 0 \\
-x_{1} & -y_{1} & -z_{1} & 1
\end{array}\right]
$$

Which $x_{1}, y_{1}, z_{1}$ is the coordinates of a point on the arbitrary axis in $\mathrm{o}_{\mathrm{m}}$-xyz coordinates, the purpose of this translation is to translate this point to $o_{s}$.

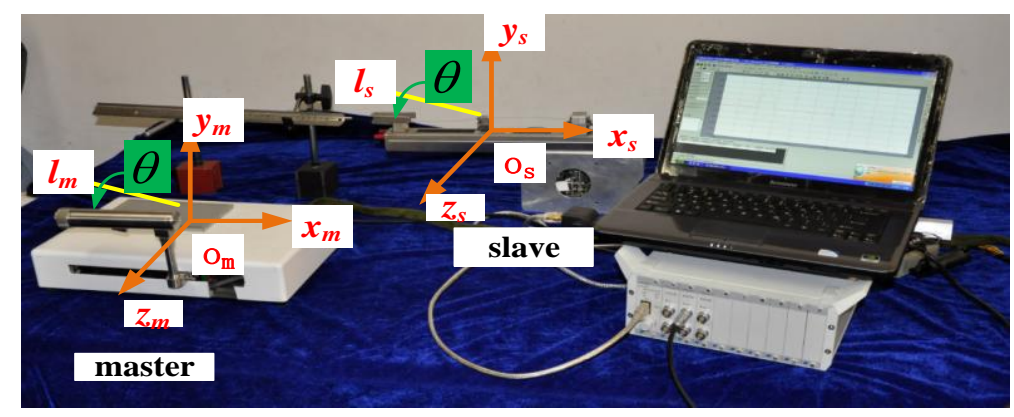

Fig. 1 Master-slave system

The rotation transformation matrix around the zs axis and the xs axis is

$$
\begin{aligned}
& T_{2}=\left[\begin{array}{cccc}
\cos \gamma & \sin \gamma & 0 & 0 \\
-\sin \gamma & \cos \gamma & 0 & 0 \\
0 & 0 & 1 & 0 \\
0 & 0 & 0 & 1
\end{array}\right] \\
& T_{3}=\left[\begin{array}{cccc}
1 & 0 & 0 & 0 \\
0 & \cos \alpha & \sin \alpha & 0 \\
0 & -\sin \alpha & \cos \alpha & 0 \\
0 & 0 & 0 & 1
\end{array}\right]
\end{aligned}
$$


At this time, the arbitrary axis $l_{\mathrm{m}}$ are coincide with the $y_{\mathrm{s}}$ axis coincide, will be around any axis of rotation into a rotation around the $y_{\mathrm{s}}$ axis, the rotation of the transformation matrix:

$$
T_{4}=\left[\begin{array}{cccc}
\cos \theta & 0 & -\sin \theta & 0 \\
0 & 1 & 0 & 0 \\
\sin \theta & 0 & \cos \theta & 0 \\
0 & 0 & 0 & 1
\end{array}\right]
$$

The inverse transformation to the rotation of the xs axis and the zs axis is

$$
\begin{aligned}
& T_{5}=\left[\begin{array}{cccc}
1 & 0 & 0 & 0 \\
0 & \cos \alpha & -\sin \alpha & 0 \\
0 & \sin \alpha & \cos \alpha & 0 \\
0 & 0 & 0 & 1
\end{array}\right] \\
& T_{6}=\left[\begin{array}{cccc}
\cos \gamma & -\sin \gamma & 0 & 0 \\
\sin \gamma & \cos \gamma & 0 & 0 \\
0 & 0 & 1 & 0 \\
0 & 0 & 0 & 1
\end{array}\right]
\end{aligned}
$$

At last, the rotation axis ls should be translate to the starting position:

$$
T_{7}=\left[\begin{array}{cccc}
1 & 0 & 0 & 0 \\
0 & 1 & 0 & 0 \\
0 & 0 & 1 & 0 \\
x_{1} & y_{1} & z_{1} & 1
\end{array}\right]
$$

Therefore, the overall transformation matrix of the whole angle mapping process is $T=T_{0} \cdot T_{1} \cdot T_{2} \cdot T_{3} \cdot T_{4} \cdot T_{5} \cdot T_{6} \cdot T_{7}$

The above for the general situation of the matrix transformation, special circumstances, if the rotation axis of the origin or the axis of a coordinate, the transformation is relatively simple.

Displacement Mapping. In order to meet the requirements of different delivery speeds, the three gears are set to achieve synchronous mobile, slow moving and fine adjustment.

1:1Synchronous Movement. Synchronous movement of the coordinate transformation can only be carried out on the coordinates of the point of arrival, the transformation matrix is the conversion matrix between the two coordinate systems.

$$
T_{0}=\left[\begin{array}{rrrr}
1 & 0 & 0 & 0 \\
0 & 1 & 0 & 0 \\
0 & 0 & 1 & 0 \\
-x_{m} & -y_{m} & -z_{m} & 1
\end{array}\right]
$$

where, $\mathrm{xm}, \mathrm{ym}, \mathrm{zm}$ respectively indicates that the origin of the master coordinate system in the coordinates of the corresponding coordinates of slave.

2:1 slow movement. The guide wire into the branch vessels, in narrow vessels within the movement may require a slower rate. Therefore, set the 2:1 slow moving files. Non synchronous mobile need to add geometric transform in zoom synchronous coordinate transformation based on transformation matrix corresponding to the slow moving 2:1 including the following steps:

First, the starting point should be translate to the point is.

$$
T_{0}=\left[\begin{array}{cccc}
1 & 0 & 0 & 0 \\
0 & 1 & 0 & 0 \\
0 & 0 & 1 & 0 \\
-x_{b m} & -y_{b m} & -z_{b m} & 1
\end{array}\right]
$$


Among them, xbm, ybm, zbm, respectively, indicates coordinates that the starting point of the slow motion in os-xyz.

Then the scaling transformation is carried out as T1.

At last, the starting point of the master, which is now moving from the position of the coordinate origin os to the starting point of the slow moving action in the os-xyz coordinate. The transformation matrix for this step is $\mathrm{T} 2$

$$
\begin{aligned}
T_{1} & =\left[\begin{array}{llll}
1 & 0 & 0 & 0 \\
0 & 1 & 0 & 0 \\
0 & 0 & 1 & 0 \\
0 & 0 & 0 & 2
\end{array}\right] \\
T_{2} & =\left[\begin{array}{cccc}
1 & 0 & 0 & 0 \\
0 & 1 & 0 & 0 \\
0 & 0 & 1 & 0 \\
x_{b s} & y_{b s} & z_{b s} & 1
\end{array}\right]
\end{aligned}
$$

Where, xbs, ybs, zbs, respectively, express the starting point coordinate from the slave coordinate os-xyz.

The total transform matrix for the slow movement is $T=T_{0} \cdot T_{1} \cdot T_{2}$.

5:1 fine adjustment. Fine adjustment is done to adjust the position of small amplitude in the guide wire close to the focus position. The mapping transformation process is same to the slow moving.

The overall transformation matrix is

$$
T=T_{0} \cdot T_{1} \cdot T_{2}=\left[\begin{array}{cccc}
1 & 0 & 0 & 0 \\
0 & 1 & 0 & 0 \\
0 & 0 & 1 & 0 \\
-x_{b m} & -y_{b m} & -z_{b m} & 1
\end{array}\right] \cdot\left[\begin{array}{cccc}
1 & 0 & 0 & 0 \\
0 & 1 & 0 & 0 \\
0 & 0 & 1 & 0 \\
0 & 0 & 0 & 5
\end{array}\right] \cdot\left[\begin{array}{cccc}
1 & 0 & 0 & 0 \\
0 & 1 & 0 & 0 \\
0 & 0 & 1 & 0 \\
x_{b s} & y_{b s} & z_{b s} & 1
\end{array}\right]
$$

\section{Conclusion}

With the aid of the master-slave hardware system of minimally invasive vascular interventional surgery robot, the action of slave effector can be obtained by master movement based on the graphics transformation. The mapping process of delivery and rotation are derived. And the derivation of the delivery action were analyzed according to different mapping speed ratio. In mapping transformation, the coordinates of the termination point of the action can be obtained only by the homogeneous coordinates of the starting point of the action. All of these work will lay the foundation for the further realization of the position control of the master-slave intervention surgery.

\section{Reference}

[1] Pisla D, Gherman B, Vaida C, et al. An active hybrid parallel robot for minimally invasive surgery Robot. Com-Int Manuf .2013: 29(4): 203-221

[2] Barbosa JABA, Barayan G, Gridley CM, et al. Parent and patient perceptions of robotic vs open urological surgery scars in children. J Urol. 2013:190(1):244-250

[3] Fine HF, Wei W, Goldman RE, Simaan N (2010) Robot-assisted ophthalmic surgery. Can J Ophthalmol .45(6):581-584.

[4] S. K. Kim, W. H. Shin, S. Y. Ko, et al. Design of a compact 5-DOF surgical robot of a spherical mechanism: CURES. In Proc. IEEE/ASME Int. Conf. Adv. Intell. Mechatron. Xi'an, China, 
Jul. 2008:990-995.

[5] M. J. H. Lum, D. C. W. Friedman, et al. The RAVEN: Design and validation of a telesurgery system. Int. J. Robot. Res., 2009:28: 1183-1197.

[6] P. Berkelman and J.Ma. A compact modular teleoperated robotic system for laparoscopic surgery. Int. J. Robot. Res., 2009:28:1198-1215.

[7] Wangsheng. Lu, Da Liu, Zengmin Tian, Dapeng Zhang. The Analysis of Key Technologies of Intravascular Intervention Surgical Robot. Journal of Biomedical Engineering Research, 2009:28(4):303-306.

[8] Zhanchuan. Zheng, Wenping Yang, Xiaohui Chen. The Application of DSA in Intervention Surgery. Journal of Youjiang Medical College for Nationalities, 2009: 31(4): 683-684.

[9] Moon Y, Choi J. Robotic Systems for Minimally Invasive Diagnosis and Therapy[M]// Biomedical Engineering: Frontier Research and Converging Technologies. 2016:463-487.

[10] Avgousti S. Medical telerobotic systems: current status and future trends[J]. BioMedical Engineering OnLine, 2016, 15(1).

[11] Kodama H, Shi C, Kojima M, et al. Catheter manipulation training system based on quantitative measurement of catheter insertion and rotation[J]. Advanced Robotics, 2014, 28(19):1321-1328(8).

[12] Yang C, Ma H, Fu M. Robot Teleoperation Technologies[M]// Advanced Technologies in Modern Robotic Applications. Springer Singapore, 2016.

[13] Aolin Tang, Qixin Cao, Hongbing Tan, et al. Motion Control of a Master-Slave Minimally Invasive Surgical Robot Based on the Hand-Eye-Coordination[J]. Computer Aided Surgery. 2016: 57-71.

[14] Marescaux J, Diana M. Robotics and Remote Surgery: Next Step[M]// Robotics in General Surgery. Springer New York, 2014: 479-484. 